\title{
Virtual Avatar Automatically Enhances Human Perspective Taking
}

\author{
Kazuya Nagamachi \\ Toyohashi University of Technology \\ Toyohashi, Japan \\ nagamachi@real.cs.tut.ac.jp
}

\author{
Sachiyo Ueda \\ Toyohashi University of Technology \\ Toyohashi, Japan \\ ueda@cs.tut.ac.jp
}

\author{
Maki Sugimoto \\ Keio University \\ Tokyo, Japan \\ sugimoto@ics.keio.ac.jp
}

\author{
Masahiko Inami \\ The University of Tokyo \\ Tokyo, Japan \\ inami@star.rcast.u-tokyo.ac.jp
}

\author{
Michiteru Kitazaki \\ Toyohashi University of Technology \\ Toyohashi, Japan \\ mich@tut.jp
}

\begin{abstract}
Reasoning about other people's mind is important to successful social communication. Visual perspective taking is the basic ability to infer others' mind, denoting the process of judgment what and how a certain object looks from the others' viewpoint. Recent research showed that the understanding of how a target appears to another person occurs spontaneously. However, the details of the process have not yet been clear. We aimed to investigate whether visual perspective taking occur in a virtual environment and how fast the process is. In an experiment, participant judged the direction of visual stimulus from the viewpoint of a humanoid avatar and responded using joystick. To know the time course of the perspective taking, we manipulated the interval time between the appearance of humanoid avatar and the target visual stimulus presentation. We found that the existence of humanoid avatar improves the performance of the visual perspective taking task in quick interval time condition. This result suggests that the visual perspective-taking is enhanced by the virtual avatar in a very fast time scale.
\end{abstract}

\section{CCS CONCEPTS}

- Human-centered computing $\rightarrow$ Virtual reality.

\section{KEYWORDS}

Perspective taking, Mental rotation, Vision, Theory of mind

\section{ACM Reference Format:}

Kazuya Nagamachi, Sachiyo Ueda, Maki Sugimoto, Masahiko Inami, and Michiteru Kitazaki. 2019. Virtual Avatar Automatically Enhances Human Perspective Taking. In The 17th International Conference on Virtual-Reality Continuum and its Applications in Industry (VRCAI '19), November 14-16, 2019, Brisbane, QLD, Australia. ACM, New York, NY, USA, 2 pages. https: //doi.org/10.1145/3359997.3365723

Permission to make digital or hard copies of part or all of this work for personal or classroom use is granted without fee provided that copies are not made or distributed for profit or commercial advantage and that copies bear this notice and the full citation on the first page. Copyrights for third-party components of this work must be honored.

For all other uses, contact the owner/author(s).

VRCAI '19, November 14-16, 2019, Brisbane, QLD, Australia

(C) 2019 Copyright held by the owner/author(s).

ACM ISBN 978-1-4503-7002-8/19/11.

https://doi.org/10.1145/3359997.3365723

\section{INTRODUCTION}

The ability to reason other people's mental states is important to make smooth communication with others. Understanding the state of another person's mind requires consideration of perspectives in the visual, conceptual, and emotional domains ('Theory of mind').In particular, visual perspective taking is a core process of social cognition. Visual perspective taking provide the insight about whether an object is or is not visible to another person (level 1perspective taking) and how that object appears to the other person (level 2 perspective taking) [Flavell et al. 1981]. Level 1 perspective taking appears very early in development compared to level 1 and appears even in other animals [Maginnity and Grace 2014]. It has been also argued that Level 1 perspective taking occurs spontaneously, whereas Level 2 does not so due to the mind reading system's limited capacity [Surtees et al. 2011].

Recently researches, however, reported that level 2 perspective taking occurs spontaneously [Elekes et al. 2016]. Neuroscientific studies also support this proposal [Böckler and Zwickel 2013]. If the proposal is correct, Level 2 perspective taking may occur very early in time. To investigate the time course of the perspective taking, this study manipulated the interval time between the appearance of humanoid avatar and the target visual stimulus presentation, and conducted visual perspective taking task. If the existence of humanoid avatar improves the performance of the perspective taking task even in quick interval time condition, it supports the possibility that the visual perspective taking occurs rapidly and spontaneously.

\section{METHODS}

\subsection{Participants}

Twenty paid volunteers (17 male, 3 female) participated in the experiment. They had healthy binocular vision. All participants provided written informed consent before the experiment. All the experiments were approved by the Ethical Committee for HumanSubject Research at Toyohashi University of Technology, and all experiments were performed in accordance with the committee's guidelines and regulations.

\subsection{Apparatus}

Visual stimuli were generated and controlled by a computer (IntelCore i7-8700, 3.20GHz, RAM 32.0GB, NVIDIA GeForceGTX1070Ti, 
MS-Windows10, Unity) and presented on a head mounted display (HTC Vive Pro:1440 x 1600 pixel, 90Hz refresh). Participants answered the task by moving a joystick.

\subsection{Stimuli and Conditions}

In the virtual space, a table was placed in the center of the room, and a chair alone or an avatar sitting on the chair were presented at one of the three positions on the left, back, and right of the table.Then, the broken ring was presented on the table. The crack of the broken ring was in one of eight directions $\left(0^{\circ}, 45^{\circ}, 90^{\circ}, 135^{\circ}, 180^{\circ}, 225^{\circ}, 270^{\circ}\right.$, $315^{\circ}$ ) like Landolt ring (Figure 1). We set two conditions for the interval time between the chair or avatar presentation and the ring presentation (Quick:200ms andSlow:1000ms). All combinations of the conditions ( 2 with/without avatar, 2 quick / slow interval time, 3 position of avatar and chair, 8 direction of the crack of the ring) were repeated twice in a random order, and they were conducted within the same block.
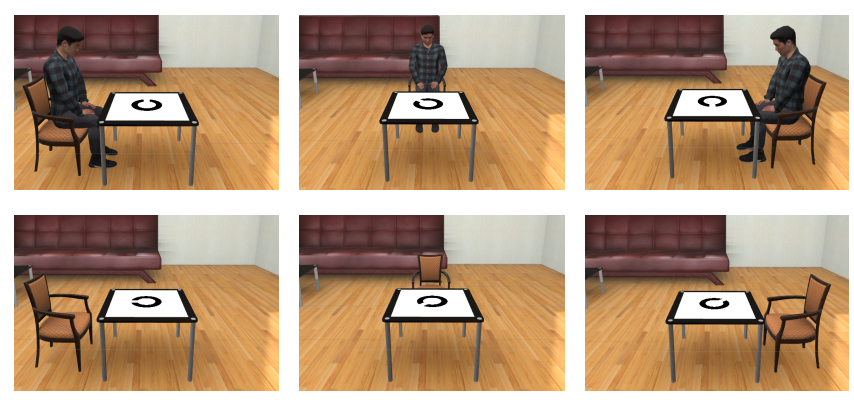

Figure 1: Examples of the stimuli of with (upper panels) and without (lower panels) human avatar conditions

\subsection{Procedures}

After fixation cross presentation, the room with a table, a chair and an avatar ("without avatar condition" is for a table and chair only)was presented. Subsequently, the broken ring was presented on the table after $200 \mathrm{~ms}$ or $1000 \mathrm{~ms}$, depending on the conditions. Participants were asked to judge the direction of the clack in the ring from an avatar's perspective and to respond with the joystick as accurately and quickly as possible.

\section{RESULTS}

For analysis, we treated the trials in which the participant moved the joystick within the range of plus or minus 22.5 degrees from the correct angle as the correct response.

To examine the effect of avatar presence on perspective taking, we conducted two-way repeated-measures ANOVA (2 with/without avatar $\times 2$ quick/slow interval) for response times of the correct trials. The different conditions of the position of avatar(chair) and the direction of the crack were merged. We found main effects of the existence of avatar $(F(1,19)=42.348, p<.0001)$, the length of interval $(\mathrm{F}(1,19)=146.976, \mathrm{p}<.001)$, and an interaction of the existence of the avatar and the length of interval $(\mathrm{F}(1,19)=4.916, \mathrm{p}=.039)$. A post-hoc test revealed that a simple main effect of the existence of avatar was significant only for the quick interval condition $(\mathrm{F}(1,19)=18.868$, $\mathrm{p}<.001)$. For the quick interval condition, the response time was faster with avatar condition than without avatar condition (Figure 2 left).

We also conducted two-way repeated-measures for error rates.We found only main effects of the existence of avatar $(F(1,19)=5.432$, $\mathrm{p}=.031)$. The main effect of the length of interval $(\mathrm{F}(1,19)=0.583$, $\mathrm{p}=.454)$ and the interaction of the existence of the avatar $\mathrm{x}$ the length of interval $(\mathrm{F}(1,19)=0.013, \mathrm{p}=.910)$ were not significant. In both of quick and slow interval conditions, error rates were smaller with avatar condition than without avatar condition(Figure 2 right).
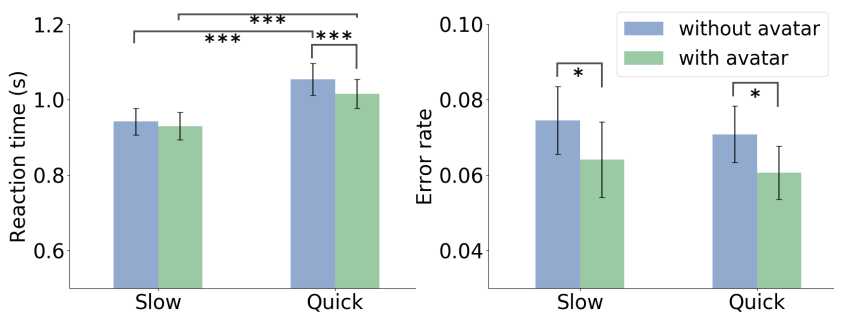

Figure 2: Results of reaction time and error rate

\section{DISCUSSION AND CONCLUSIONS}

We found that the existence of human avatar facilitates to judge the direction of visual stimuli from a different point of view than our own. The facilitate effect was shown only in quick interval condition. This result suggests that the perspective-taking of humanoid avatar occurs in a very fast time scale. The long interval would be enough to image the appearance from different viewpoints cognitively so that the time advantage of the perspective-taking of the human avatar might disappear. However, the error rates were lower in 'with avatar condition' irrespective of the length of the interval, suggesting that the fast reaction time in 'without avatar' condition might be due to the trade-off with the error rate.

\section{ACKNOWLEDGMENTS}

This research was supported by JST ERATO Grant Number JPMJER1701 (Inami JIZAI Body Project).

\section{REFERENCES}

Anne Böckler and Jan Zwickel. 2013. Influences of spontaneous perspective taking on spatial and identity processing of faces. Social Cognitive and Affective Neuroscience 8, 7 (Oct 2013), 735-740. https://doi.org/10.1093/scan/nss061

Fruzsina Elekes, Máté Varga, and Ildikó Király. 2016. Evidence for spontaneous level-2 perspective taking in adults. Consciousness and Cognition 41 (Apr 2016), 93-103. https://doi.org/10.1016/j.concog.2016.02.010

John H. Flavell, Barbara A. Everett, Karen Croft, and Eleanor R. Flavell. 1981. Young children's knowledge about visual perception: Further evidence for the Level 1Level 2 distinction. Developmental Psychology 17, 1 (Jan 1981), 99-103. https: //doi.org/10.1037/0012-1649.17.1.99

Michelle E. Maginnity and Randolph C. Grace. 2014. Visual perspective taking by dogs (Canis familiaris) in a Guesser-Knower task: evidence for a canine theory of mind? Animal Cognition 17, 6 (Nov 2014), 1375-1392. https://doi.org/10.1007/s10071-0140773-9

Andrew D. R. Surtees, Stephen A. Butterfill, and Ian A. Apperly. 2011. Direct and indirect measures of Level-2 perspective-taking in children and adults. British fournal of Developmental Psychology 30 (Oct 2011), 75-86. https://doi.org/10.1111/ j.2044-835X.2011.02063.x 